\title{
Research Paper \\ Content and Citation Analysis of Articles Published in Iranian Journal of Aging, 2006-2015
}

\author{
Yadollah Abolfathi Momtaz ${ }^{1,2}$, Ahmad Delbari ${ }^{1,3},{ }^{*}$ Robab Sahaf $^{1}$, Shahla Roustaei ${ }^{1}$, Raziye Sadat Hosseini ${ }^{1}$, Maryam \\ Ramshini $^{4}$
}

1. Department of Ageing, Iranian Research Center on Aging, University of Social Welfare and Rehabilitation Sciences, Tehran, Iran.

2. Institute of Gerontology, Universiti Putra, Selangor, Malaysia.

3. Department of Neurobiology and Care Sciences and Society, Karolinska Institute, Solna, Sweden.

4. Department of Psychology and Education of Exceptional Children, Faculty of Humanities and Social Sciences, Science and Research Branch, Islamic Azad University, Tehran, Iran.

Citation: Abolfathi Momtaz Y, Delbari A, Sahaf R, Roustaei Sh, Hosseini RS, Ramshini M. [Content and Citation Analysis of Articles Published in Iranian Journal of Aging, 2006-2015 (Persian)]. Iranian Journal of Ageing. 2017; 12(2):208-219. http://dx.doi.org/10.21859/sija-1202208

http://dx.doi.org/10.21859/sija-1202208

Received: 17 Jan. 2017

Accepted: 21 Apr. 2017

Key words:

Citation analysis,

Content analysis,

Iranian Journal of

Ageing

\section{A B STRACT}

Objectives Scientific journals are one of the most important tools for knowledge transition and translation in the word. Therefore, it is necessary to evaluate these journals with the aim to control the research published in them. The study aimed to conduct a content and citation assessment of articles published in the Iranian Journal of Ageing.

Methods \& Materials A descriptive bibliometric analysis design was employed in this study. All available manuscripts published from 2006 through 2015 were reviewed. A valid researcher instrument was constructed and employed for data collection. SPSS version 23 was used for analysis.

Results Of the 298 published manuscripts, the most common field of publication were physical activity with $18.5 \%$, followed by mental and physical area with $12.4 \%$ and $10.4 \%$, respectively. The most popular method used in these published papers (67\%) was descriptive-analytic, and the least method (7.1\%) was clinical trials. The analysis of the geographical distribution of published papers revealed that the majority of the papers originated from Tehran, but no articles were published from the five provinces of North Khorasan, Kurdistan, Kohgiluyeh \& Boyer-Ahmad, Hormozgan, and Hamedan. The average of editorial time (speed from submission to publication) was 284 days (SD=143). Approximately $22 \%$ of the papers were cited at least once. The mean number of citations per document was $2.26(\mathrm{SD}=1.95)$. Results of the inferential statistics revealed that research setting and published time were significantly associated with citation.

Conclusion According to the findings from the present study, in order to improve the quantity and quality of the Journal, it is suggested that the editorial board of the Journal take up measures to reduce editorial time. It is also suggested that they should focus on the scope of the Journal and consider appropriate geographical distribution of the published papers.

\section{* Corresponding Author:}

Robab Sahaf, PhD

Address: Department of Ageing, Iranian Research Center on Aging, University of Social Welfare and Rehabilitation Sciences, Tehran, Iran.

Tel: +98 (21) 22180154

E-mail: robabsahaf@gmail.com 


\section{Extended Abstract}

\section{Objectives}

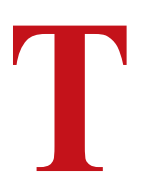

oday, a community is considered as developed if it is scientifically prominent along with highlighted economic and demographic indicators. The spread of science and its development are requirements for the scientific development of any society, and they are one of the most prestigious indicators of scientific status. This is the reason journals and quarterly newspapers and scientific publications are the main forms of knowledge transfer at the level of science and research that have been the focus of attention in international communities [1,2]. Therefore, given the great role and importance of scientific journals in the field of science and knowledge, it is necessary for them to be evaluated periodically, so as to help policy makers in the field of science and knowledge of the country in the planning of research policies, assessing and controlling the progress made and promoting the quality of research activities.

\section{Methods \& Materials}

This descriptive study was performed by citation method. Using this evaluation method, we can make correlations between different content variables and other research variables, discussion of citation and the amount of scientific referral to oneself and others, and the relationship between a work and the article cited in the index [3, 4]. The target research community of all articles published in Iran's Elderly Science Journal and sample size include all articles published in this Journal during the years 20152016 with a total of 298 articles as conducted by census.

The tool used in this research is a content and citation analysis checklist. It was made by the researcher, whose narrative content was confirmed after approval of the plan by studying various papers in the field, holding meetings on the topic of the questionnaire in the presence of professors and specialists in the field. Then the questionnaire was given to five university experts for performing narrative validity analysis, which included articles on the profile of the authors, areas of research, the method used in the articles, place of research, duration of receipt until the paper is published, and citation rates. After verifying the questionnaire, upon obtaining a university license (Plan number 1535) and in collaboration with the Elderly Science Journal for accessing to the website of scientific-research quarterly of the elderly, each published article was investigated during the period of 2015-2016 and articles that could not be achieved with full text were excluded from the research. The required data were accordingly collected and statistically analyzed. For data analysis, descriptive and analytical statistics were employed using SPSS 23.

\section{Results}

According to research findings, duration of publication of articles in 2013 was an average of 365.19 days, which is the longest time, and its minimum duration in 2015 was an average of 213 days. In terms of the research method used in the articles by the authors, it must be admitted that $43 \%$ of articles used descriptive-analytical method, which is the most used method and $7.1 \%$ of the articles used the clinical trial method, which is the least used method. Semi-experimental method was used in $25.5 \%$ articles and descriptive method in $18.1 \%$. Two qualitative and review methods are used in a similar number of articles (6\%).

What has been obtained from the dispersal of the research subject areas in published articles is that the mostly used research area is the field of "sports" with $18.46 \%$, and the least is the field of "devotees," which did not reach $1 \%$. Of course, in some research areas, the same percentage was observed, for example, in the field of "Social Issues" and "Instrumentation" (5.3\%), in two areas of "drug problems" and "economy" (2.35\%), in the research areas of the "Senior Services System", "Health Education", "Elderly patients", "Elderly biology" and "careers" $(2.0 \%)$, and in the categories of "Elderly" and "Accidents" (1.01).

With respect to the dispersion of research sites, we must consider that Tehran (58.9\%) is among the cities which have the largest number of articles submitted to the Journal and Isfahan and Fars ranked second (1.7\%) and third (1.5\%) respectively. Cities of Khorasan Razavi, Chaharmahal Bakhtiari, and East Azarbaijan account for almost $4 \%$ of the articles. Also most other cities, including Mazandaran, South Khorasan, Golestan, Ilam, Kermanshah, East Azarbaijan, Qom, Yazd, and Gilan, had less than 1\% of the share of articles sent to the magazine. Given the frequency of articles published in each province regarding the elderly contribution, it should also be said that among different provinces, Tehran has the largest number of published articles (56.9\%) proportional to its old age share (17.3\%).

There are 5 provinces (North Khorasan, Kurdistan, Kohgiluyeh \& Boyer-Ahmad, Hormozgan, Hamedan), which despite having different shares of the elderly people, had no published research papers; this has been partially specified in the table below. Of course, the end result is that there was a significant relationship between the num- 
ber of references to articles and factors such as different courses and place of research. Concerning the relationship between different courses and citation rates, it can be said that among all the published articles in this period, 66 articles were cited, among which 54\% were cited once, $15.2 \%$ were cited twice, $9.1 \%$ were cited three times, and the rest of the articles had four or more citations. In fact, the Chi-square test showed that there was a significant relationship between different courses in the elderly Journal and number of citations, and this relationship is in a way that articles published in years 2006 to 2011 were cited more than those in 2011 and later, and after 2011, there was no citation to the articles $\left(\mathrm{X}^{2}(9)=108.40, \mathrm{P}<0.001\right)$.

In relation to the relationship between the place of research and citation rates, it must be said that most citations have been allocated to society-based studies compared to the hospital and nursing home studies such that societybased studies (27\%) have been allocated more citation than the hospital $(21.2 \%)$ and the nursing home $(10.9 \%)$ studies $\left(\mathrm{X}^{2}(2)=7.01, \mathrm{P}<0.05\right)$.

\section{Conclusion}

Since the goal of an elderly research journal is improving knowledge level and presenting research achievements in various aging fields from all over Iran in the shortest possible time, practitioners of the Journal have to deal with all subjects of aging in different areas including biology, sociology, rehabilitation, medicine, paramedic, and psychology. They also have to accept articles from all over Iran in the right time period and take into account more or less the same proportion of articles based on results of this study.

\section{Acknowledgments}

University of Social Welfare and Rehabilitation Sciences of Tehran has financially supported the present paper.

\section{Conflict of Interest}

All authors certify that this manuscript has neither been published in whole nor in part nor being considered for publication elsewhere. The authors have no conflicts of interest to declare. 


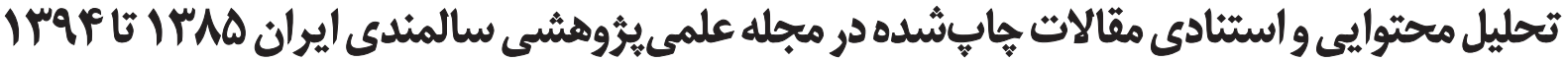

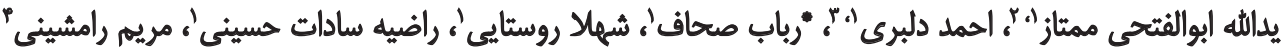 \\ | - كروه سالمندى، مركز تحقيقات سالمندي، دانشكاه علوم بهزيستى و توانبخشى، تهراني، ايران.

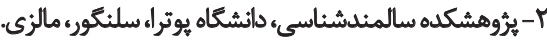

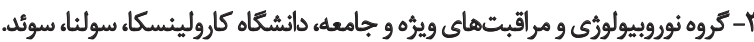

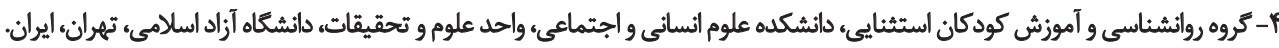

\section{حكيد}

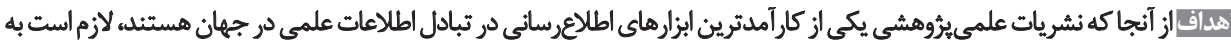

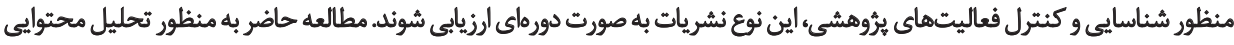

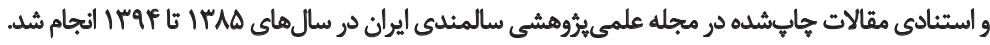

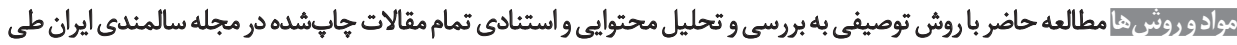

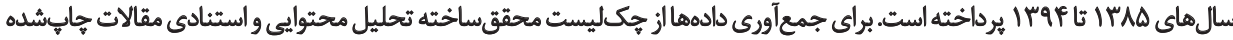

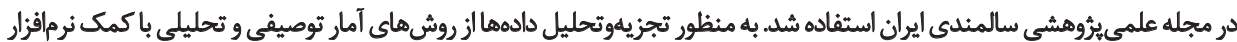
SPSS

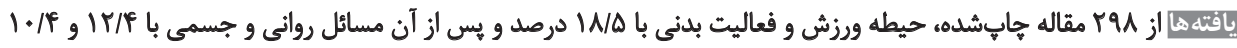

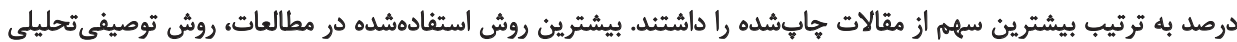

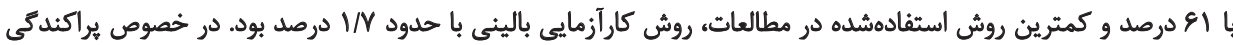

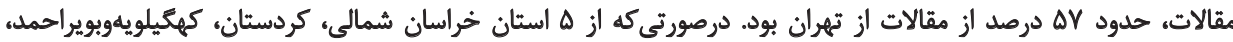

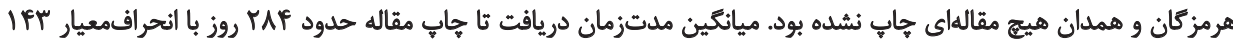

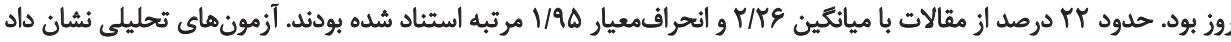

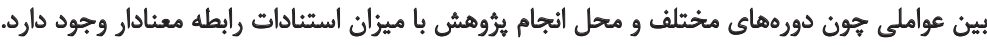

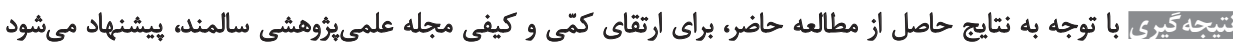

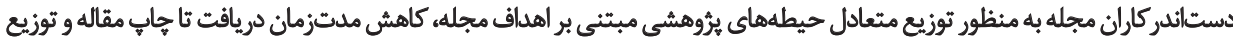
مئاسب يراكندكى جغرافيايى مقالات اقداماتى انجام دهند.

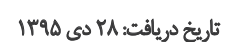

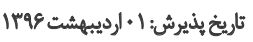

كليدواثرها:

تحليل استنادى، تحليل

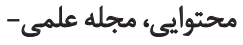
يُروهشى سالمند مجلد علدي

فصلنامها و نشريات علمى كشور از شاخصهاى ارزيابى جايكاه

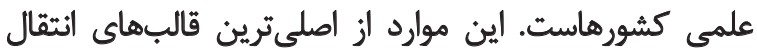

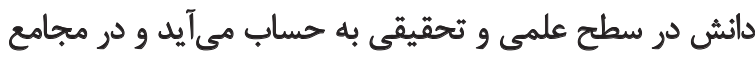

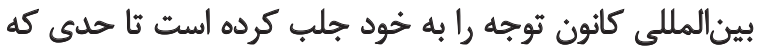

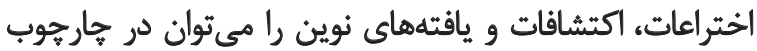

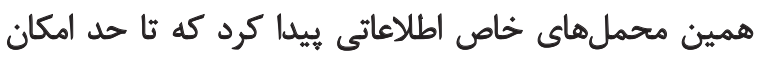

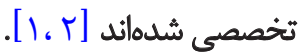

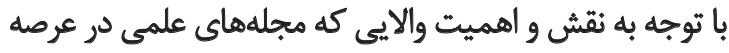

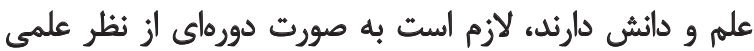

مقدمه

در دنياى امروز جامعهايى را توسعهيافته مي دانئد كه در كنار

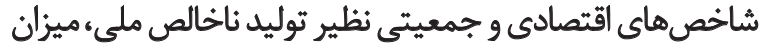

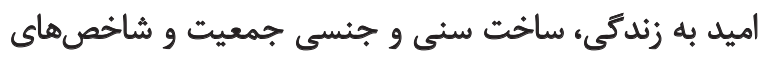

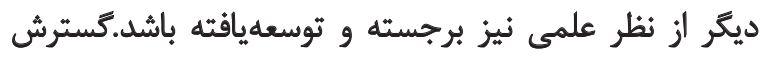

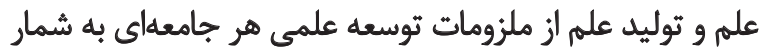

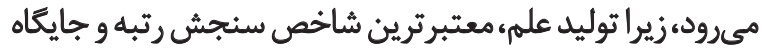

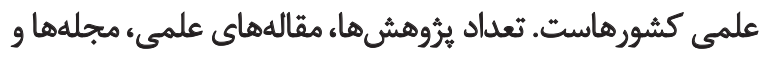


فصلنامه سالمند، نشريه مركز تحقيقات سالمندى دانشعاه

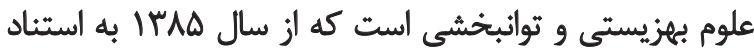

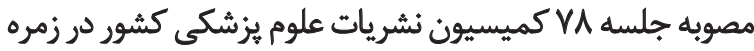

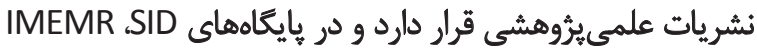

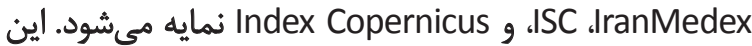

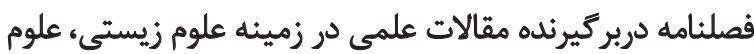

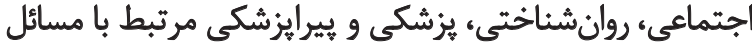

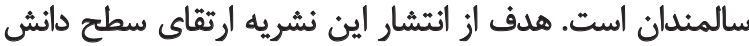

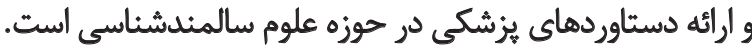

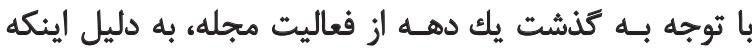

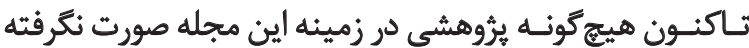

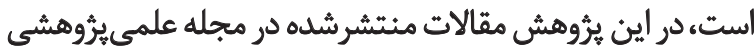

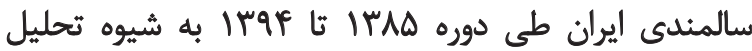

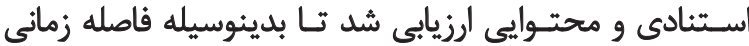

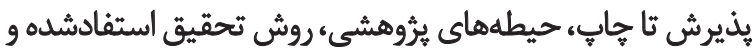

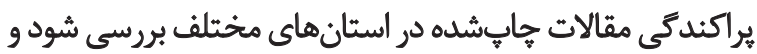

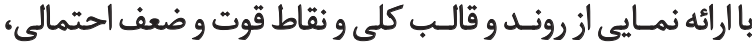
مجله را در راه رسيدن به اهداف يارى كند.

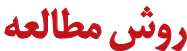

اين مطالعه توصيفى به روش استنادى انجام شد. با استفاده

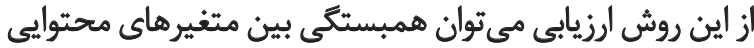

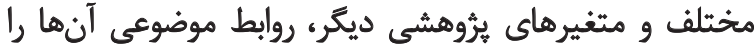

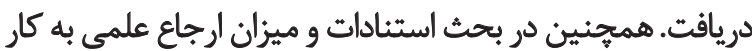
خود و ديكران مي توان به وجود رابطه بين يك اثر و و مقالهاي كه

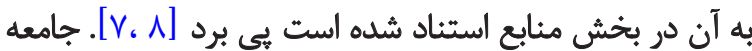

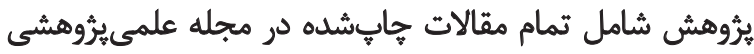

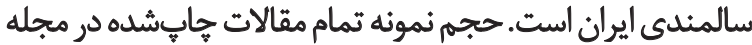

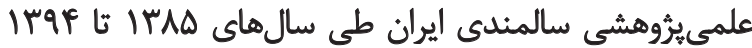

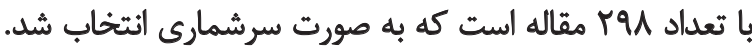

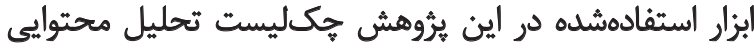

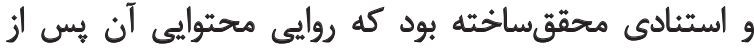

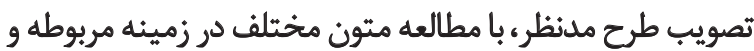

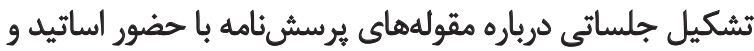

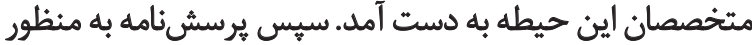

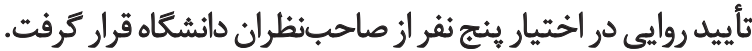
اين برسش نامه شامل مقولاتى در زمينه مشخصات نويسندكان، حيطههاى يُروهش، روش استفادمشده در مقالات، محات محل انجام

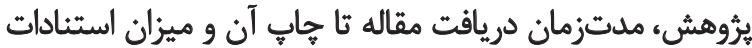

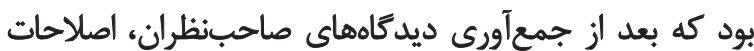

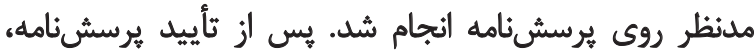

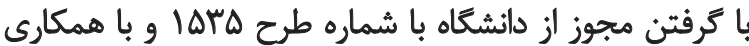

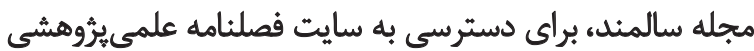

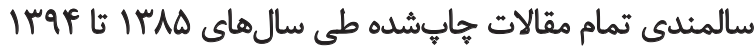

ارزيابي شوند، جراكه ارزيابى دورهاي مجلات علمىيثروهشي

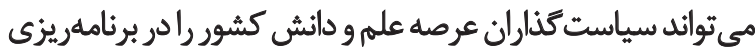

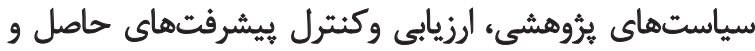

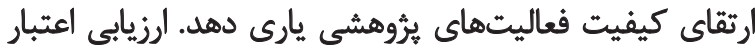

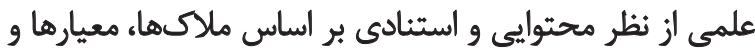

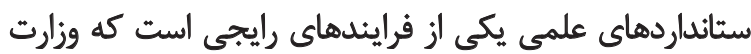

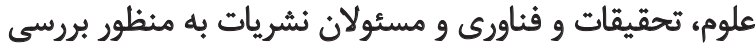

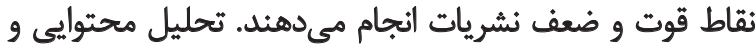

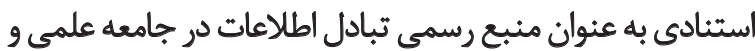

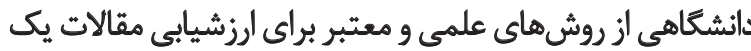

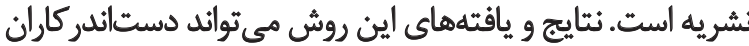

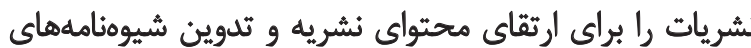

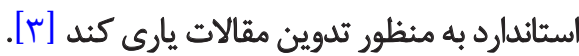

بسيارى از نشريات و مجلات در ايران وخارج از ايران با استفاده

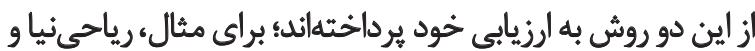

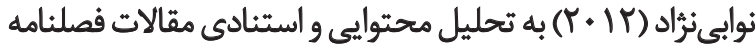

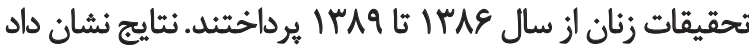

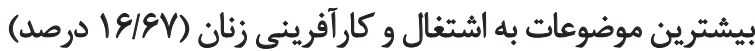

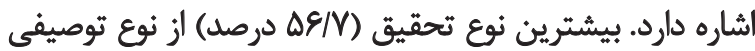

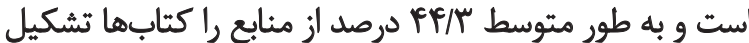

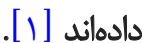

بابالحوائجى و يزديانراد در تحليل محتوايى مقالات مجلات

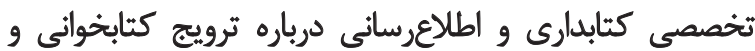

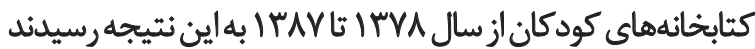

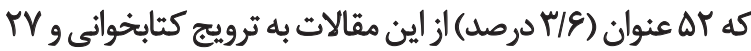

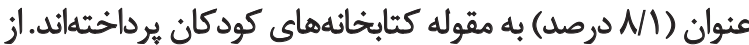

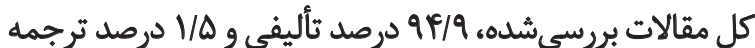

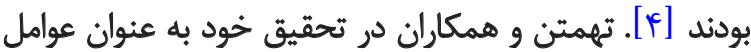

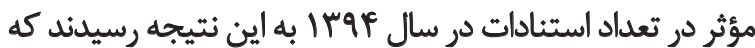

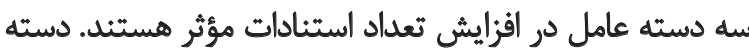

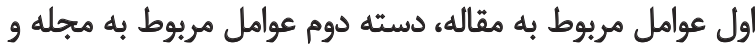
دسته سوم عوامل مربوط به نويسندكان است [هـ]

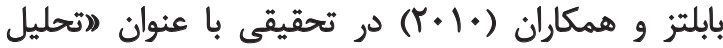

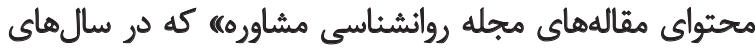

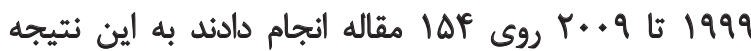

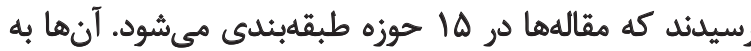

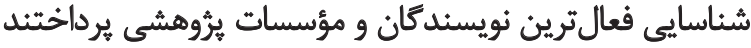

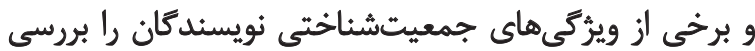

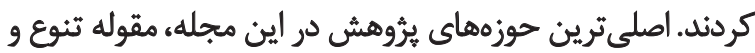

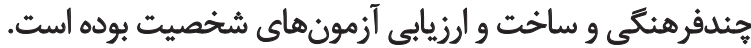

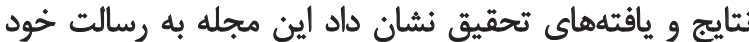

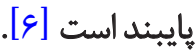




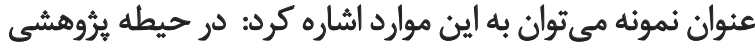

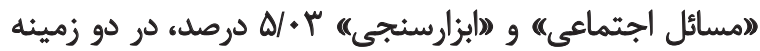

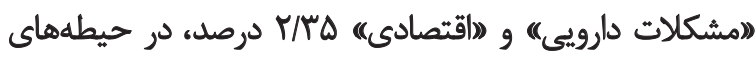

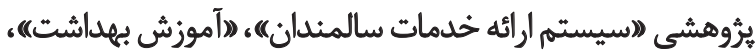

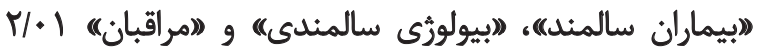

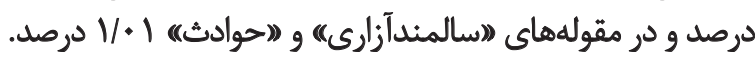
درباره يراكندكى محل انجام يرؤهش در مقايسه باسههم سالمندان

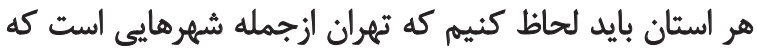

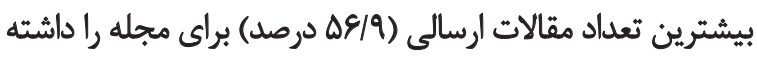

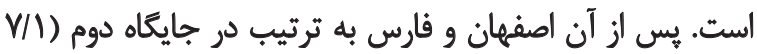

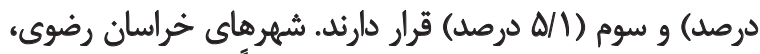

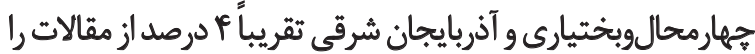

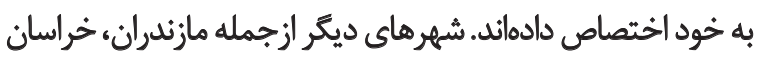

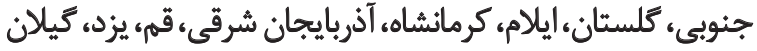
و ... كمتر از ا درصد مقاله براى مجله فرستاداناند.

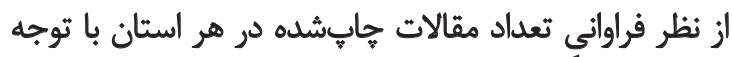

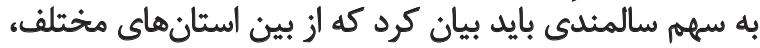

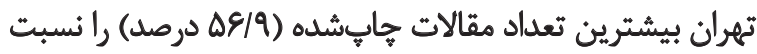

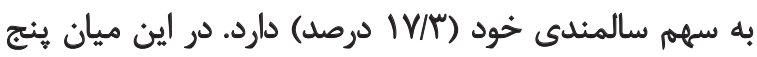

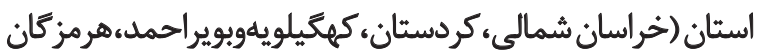

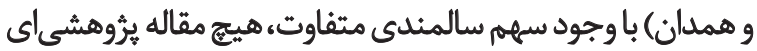
در اين زمينه ندارند (جدول شماره عأ).
بررسى شدند و مقالاتى كه دستيابى به متن كامل آنها ميسر

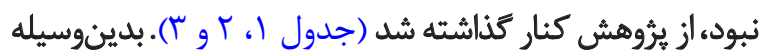

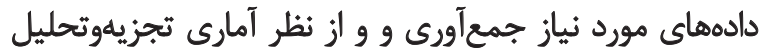

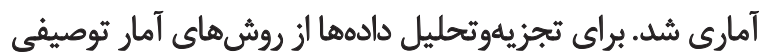

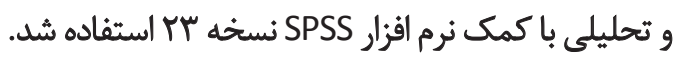

Ldịl

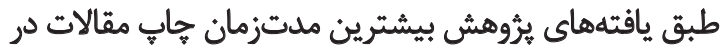

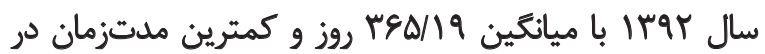

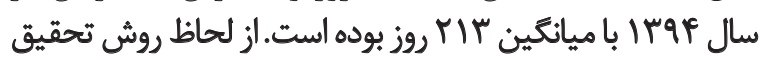

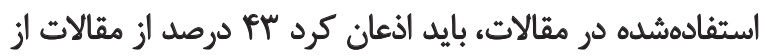

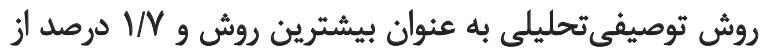

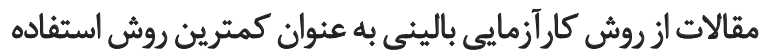

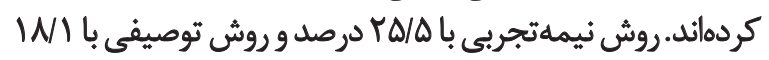

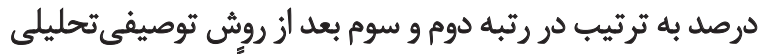

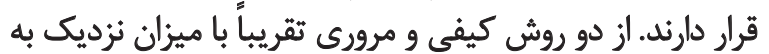
هم (و درصد) استفاده شده است.

آنجه از يراكندكى حيطههاى موضوعى يُروهش در مر مقالات

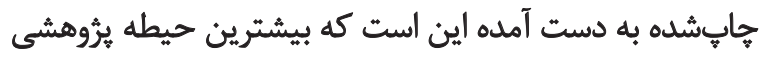

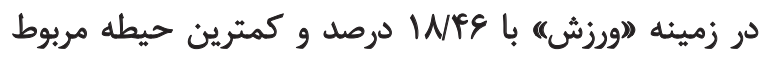

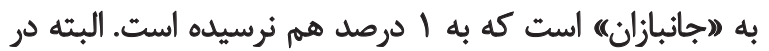

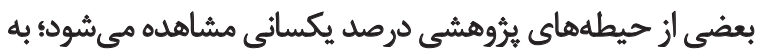

جدول 1. وضعيت مدتزمان يذيرش در سال هاى مختلف

\begin{tabular}{|c|c|c|c|}
\hline مياتعَين مدثزمان & بيشترين زمان & كمترين زمان & سال يذيرش مثالات \\
\hline rer/W & vel & so & $|r q|$ \\
\hline$r E \Delta / 19$ & $9 x q$ & $1 .+$ & Irq9 \\
\hline$m+/{ }_{1}$ & $\operatorname{sen}$ & $\varepsilon$ & (T) \\
\hline rir & PAq & 19 & Iraf \\
\hline rAY/Q & vel & $\varepsilon$ & 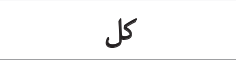 \\
\hline
\end{tabular}

L

جدول †. وضعيت روش تحقيق استمادهشه در مقالات

\begin{tabular}{|c|c|c|}
\hline درصد & فراوانى & نوع مطالعه \\
\hline$|N|$ & $\Delta f$ & توصيفى \\
\hline $\mathrm{rr} /$ & IrA & توصيفىتحليلى \\
\hline $1 / V$ & ه & كارآزمايى بالينى \\
\hline$\Delta / N$ & IV & كيفى \\
\hline 91. & $M$ & هروs \\
\hline$r \Delta / \Delta$ & ve & نيمهنجريى \\
\hline
\end{tabular}

L 


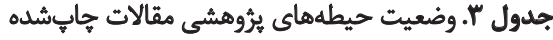

\begin{tabular}{|c|c|c|}
\hline درصد & فراوانى & حيطه بيروهش \\
\hline WPE & $\Delta \Delta$ & ورزش \\
\hline IT/RT & r & مسائل روائي \\
\hline 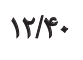 & m & مسائل جسمى \\
\hline V.๕ & iV & كيفيت زندكى \\
\hline$\Delta / V$ & IV & سلامت سالمندان \\
\hline$\Delta /+r$ & 10 & مسائل اجتماعى \\
\hline$\Delta /+r$ & 10 & ايزارسنجى \\
\hline$r / 89$ & 11 & سقوط \\
\hline r/me & 1. & تثغليه \\
\hline$r / . r$ & 9 & اوقات فراعتت \\
\hline$r / \& A$ & $\wedge$ & هنهيى \\
\hline$r / r \Delta$ & $\checkmark$ & مشكلات دارويى \\
\hline$r / r \Delta$ & $\checkmark$ & اقتصادي \\
\hline$r / \cdot 1$ & 8 & سيستم ارائه خدمات سالمندان \\
\hline$r / \omega 1$ & 8 & آموزش بهداشت \\
\hline$r / * 1$ & 8 & بيماران سالمند \\
\hline$r / .1$ & 8 & بيولوزى سالمندى \\
\hline$r / .1$ & 8 & مراقبان \\
\hline $1 / \cdot 1$ & r & سالمندآزالرى \\
\hline$V / 1$ & r & حوادث \\
\hline.$/ g V$ & r & شير دوستدار سالمند \\
\hline.$/ \& V$ & $r$ & سوء مصرف مواد \\
\hline $\operatorname{teV}$ & $r$ & سبك زندكى \\
\hline $.19 V$ & r & نظريههاى سالمندى \\
\hline $\log$ & r & آى تى و سالمندى \\
\hline.$/ F V$ & r & نيازسنجى \\
\hline$\cdot / \pi F$ & 1 & جاثبازان \\
\hline $1 .$. & ral & كل \\
\hline
\end{tabular}

닌

دورههاى مختلف و ميزان استناد به مقالات بايد بيان كرد كه

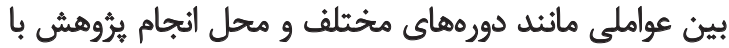

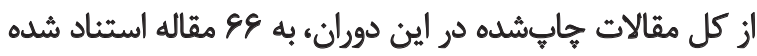

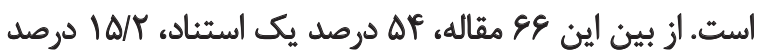


جدول F. توزيع فراوانى و درصد مقالات جابششده در مقايسه با سهم سالمندى هر استان

\begin{tabular}{|c|c|c|c|}
\hline سهرم سالمندى استان & خابشده هر استان & قرابشده هر الستان مقالات & استان \\
\hline $\mid V / r$ & $\Delta \& / q$ & iff & تهران \\
\hline$V / T^{\circ}$ & $V / I$ & $M$ & اصفهان \\
\hline$\varepsilon$ & $\Delta / 1$ & ir & فارس \\
\hline 1 & $\%$ & 1. & خراسان رضوى \\
\hline $1 / 1$ & $r / 8$ & 9 & جهارمحالوبختيارى \\
\hline$s / r$ & $m / 8$ & 9 & أذربايجان شرقى \\
\hline$r / T^{2}$ & $r / e$ & $\varepsilon$ & مركزى \\
\hline $1 / 8$ & $r /$. & $\Delta$ & قزوين \\
\hline$r / V$ & $r /$ & $\Delta$ & 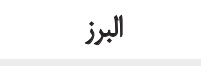 \\
\hline$r / r$ & $1 / 8$ & $r$ & خوزستان \\
\hline$r / r$ & $1 / 8$ & f & كرمان \\
\hline.$/ 9$ & $1 / 4$ & r & بوشهر \\
\hline 1 & $1 / r$ & $r$ & سمنان \\
\hline $1 / 1$ & $\cdot / A$ & r & مازنئران \\
\hline $1 / 1$ &.$/ A$ & r & خراسان جنوبيى \\
\hline $1 / 9$ & $\cdot / A$ & r & كلسثان \\
\hline .18 & $\cdot / 1$ & $r$ & ايلام \\
\hline$r / 8$ & $\cdot \mid A$ & $r$ & كرمائشاه \\
\hline$r / q$ & $\cdot / 1$ & $r$ & آَّربايايجان غربى \\
\hline $1 / 9$ &.$/ A$ & $r$ & سيستانوبلوجستان \\
\hline $1 / \wedge$ &.$/ f$ & 1 & اردييل \\
\hline $1 / \pi$ & $\cdot / r^{\circ}$ & 1 & قم \\
\hline$r / T$ &.$/ 4$ & 1 & لريشان \\
\hline$P / V$ & $\cdot / r^{\circ}$ & 1 & ي تيان \\
\hline $1 / \Delta$ &.$/ 4$ & 1 & يزد \\
\hline $1 / 0$ &.$/ p$ & 1 & زنجان \\
\hline$M$ & .1 & - & خراسان شمالى \\
\hline $1 / 9$ & $\%$ & - & كردستان \\
\hline$\cdot N$ & .1 & - & كهكلويهوبويراحمد \\
\hline $1 / 4$ & $\%$ & - & هرمزكان \\
\hline$r / 1$ &.$\%$ & • & همان \\
\hline
\end{tabular}


در ارتباط با يراكندكى حيطههاي يروهشى بايد كَت كه باوجود بردي

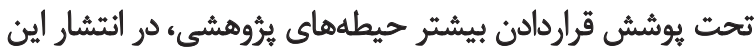

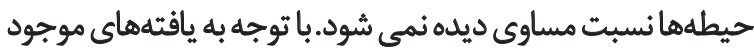

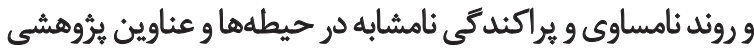

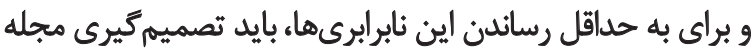

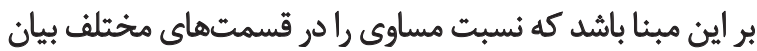

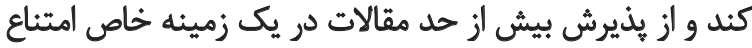

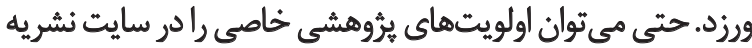

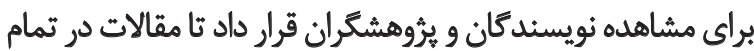
حيطهها يراكند مكى يكسانى داشته باشند.

با توجه به اينكه در رشته سالمندى دانشجو يذيرش مي شيود،

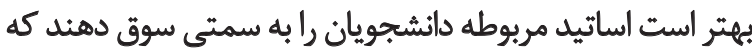

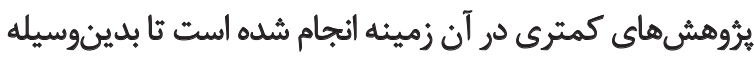

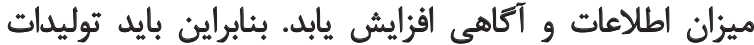

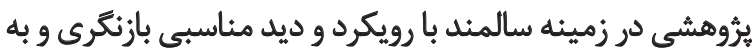

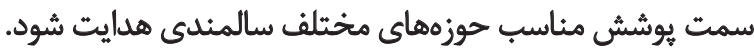

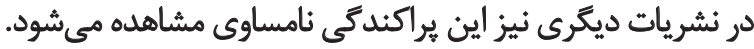

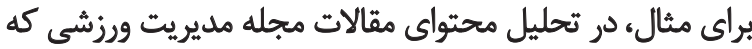

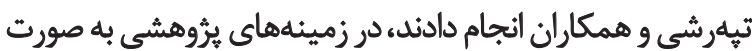

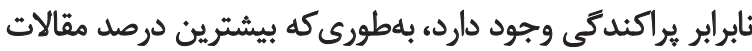

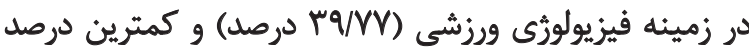

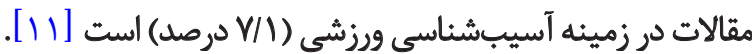

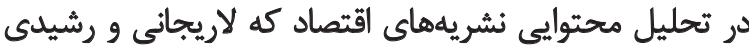

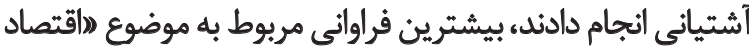

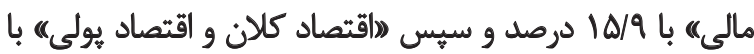

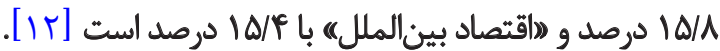

در بررسى دورههاى ده ساله از لحاظ يراكندكى استانها

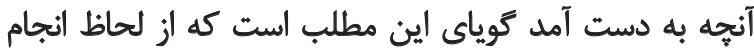

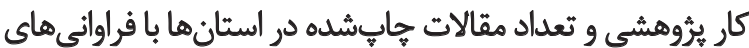

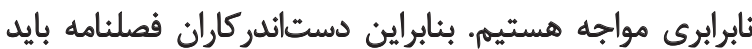

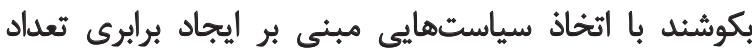

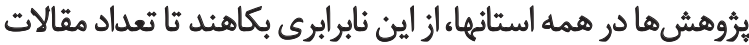

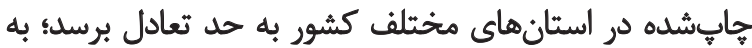

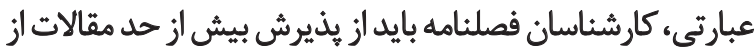

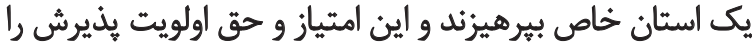

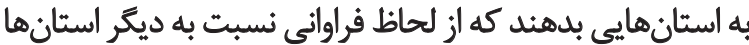

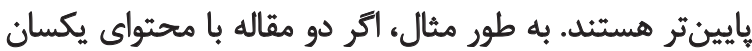

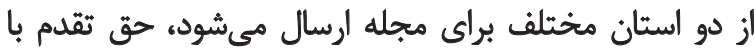

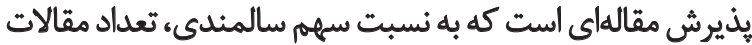

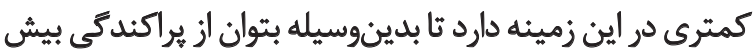

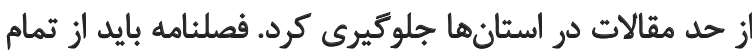
استانهاى ايران مقالات و كارهاى بروهشى داني داشته باشد. در اين يرُوهش بين دورههاي مختلف و محل انجام برؤهش با
دو استناد و // درصد سه استناد و بقيه مقالات نيز ههار استناد و

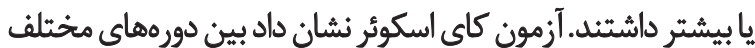

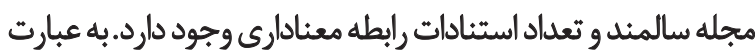

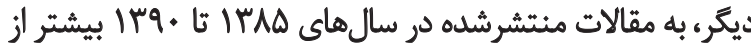

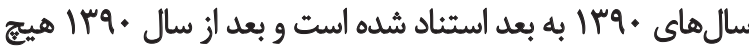

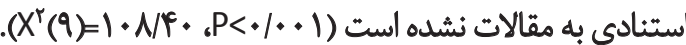

در رابطه با ارتباط بين محل انجام يُؤهش و ميزان استناد

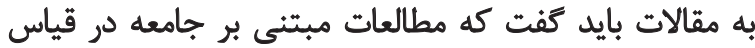

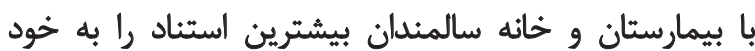

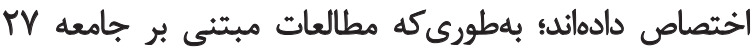

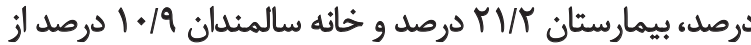

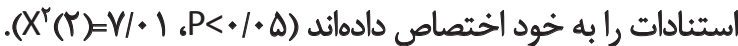

ث?

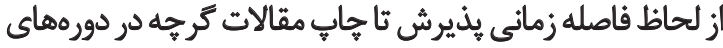

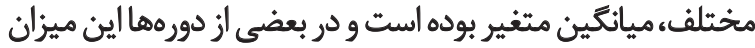

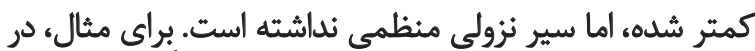

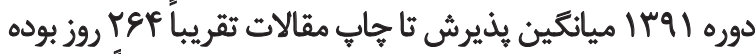

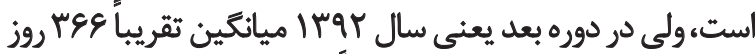

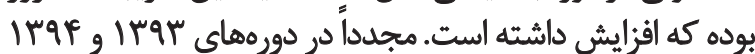

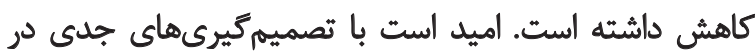

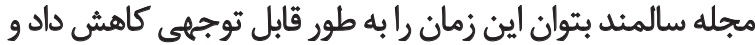

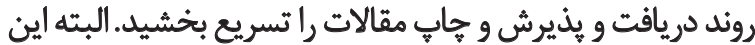
امر همكارى دستاندر كاران فصلنامه سالمند رامى رطلبد

يافتهاي تحقيق بيانكر تمركز يُروهشكُان روى روش

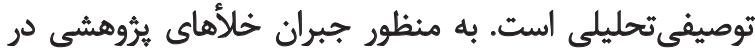

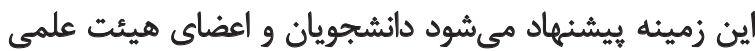

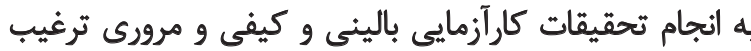

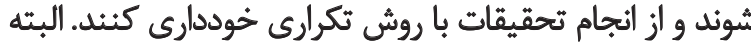

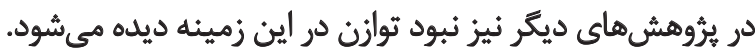

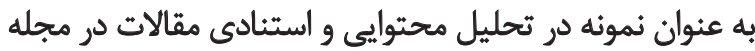

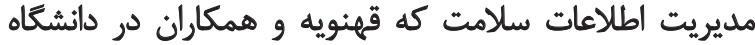

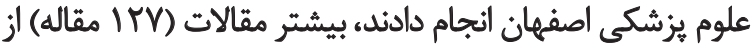

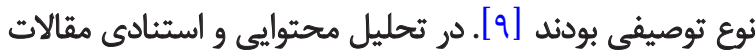

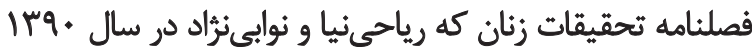

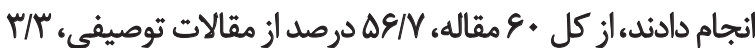

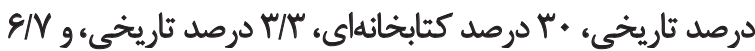

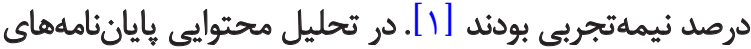

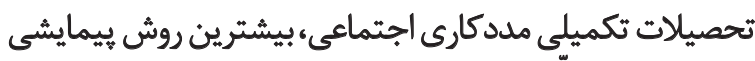

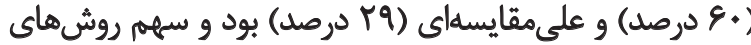

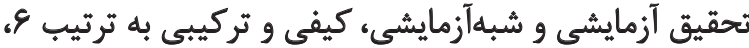

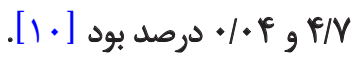


ميزان استنادات به مقالات رابطه معنادار مشاهده شد. به عبارت

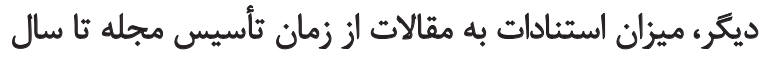

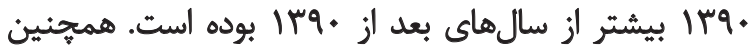

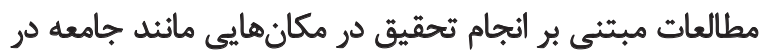

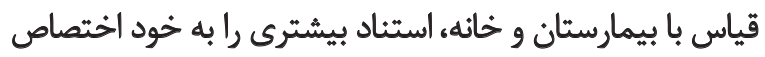

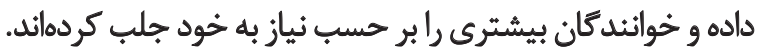

\section{نتيجليَيرى نهايى}

از آنجايى كه هدف فصلنامه علمىئروهشى سالمند ارتقاى

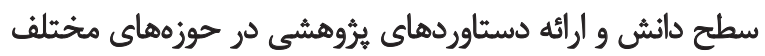

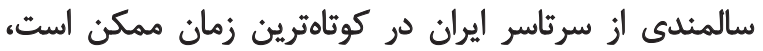

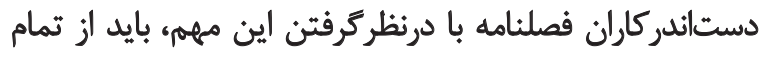

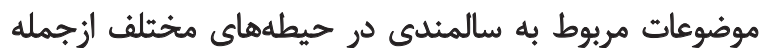

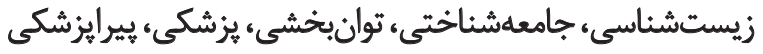

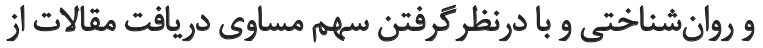

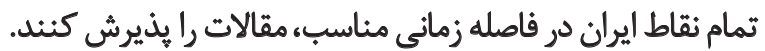

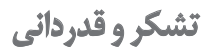

دانشكاه علوم بهزيستى و توانبخشى از اين طرح حمايت مالى كرده

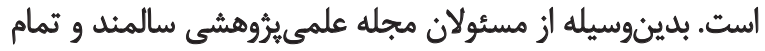

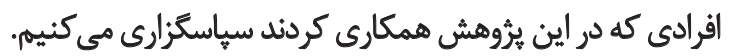




\section{References}

[1] Riyahiniya N, Navabinezhad Sh. [Content and citation analye sis of Journal of Researches of Women (Persian)]. Journal of Researches of Women. 2012; 5(1):1-13.

[2] Rezayat G. [Citation and content analysis of published articles in the journal of research in Islamic education issues (Persian)]. Research in Islamic Education Issues. 2016; 26:105-38.

[3] Zareii E, Sediqi Z. [Citation analysis of the articles published in Quarterly of Islamic Revolution Studies from 2008 to 2013 (Persian)]. Quarterly of Islamic Revolution Studies. 2014; 10(35):193.

[4] Babolhavaeji F, Yazdianrad M. [Content analysis of papers in specialized Journals of Library and Information Science regarding promotion of reading and children's libraries during 1999-2008 (Persian)]. Research on Information Science \& Public Libraries. 2011; 16(63):31-53.

[5] Allahyari MS, Daghighi Masouleh Z, Eftekhari H. [Content analysis of scientific articles in Agricultural Extension and Education Science in Iran (2009-2013) (Persian)]. Iranian Agricultural Extension and Education Journal. 2015; 11(1):229-49.

[6] Buboltz W, Deemer E, Hoffmann R. Content analysis of the Journal of Counseling Psychology: Buboltz, Miller, and Williams (1999) 11 years later. Journal of Counseling Psychology. 2010; 57(3):368-75. doi: 10.1037/a0020028

[7] Delavar A. [Research methodology in psychology and education (Persian)]. Tehran: Virayesh Publication Institute; 2005.

[8] Zarei I, Khodadad-Shahri N. [Content and citation analysis of language Related Research Journal (No. 1-21) published during 2010-2014 (Persian)]. Language Related Research. 2015; 6(4):23745

[9] Ghahnaviyeh H, Movahedi F, Yarmohamadian MH, Ajami S. [Content and citation analysis of articles published in the Journal of Health Information Management (Persian)]. Journal of Health Information Management. 2011; 8(1):82-92.

[10] Mohammadi E. [Content analysis of graduate thesis in social work (Persian)]. Journal of Social Work. 2015; 1(1):137-72.

[11] Taperashi G, Haghighi S, Abdolvahab Z. [Content analysis of sports management articles in scientific magazines (Persian)]. Communication Management in Sports Media. 2013; 1(1):53-60.

[12] Hassan Larijani H, Rashidi Ashtiyani A. [Content analysis of academic-scholarly journals in the field of economics published during 2006-2010 (Persian)]. The Journal of Planning and Budgeting. 2011; 16(3):133-57. 
